\title{
Stochastic processes in magnetization reversal involving domain wall motion in magnetic memory elements
}

\author{
Paul Bouquin, ${ }^{1,2}$ Joo-Von Kim, ${ }^{1}$ Olivier Bultynck, ${ }^{2}$ Siddharth Rao, ${ }^{2}$ \\ Sebastien Couet, ${ }^{2}$ Gouri Sankar Kar, ${ }^{2}$ and Thibaut Devolder ${ }^{1, *}$ \\ ${ }^{1}$ Université Paris-Saclay, CNRS, Centre de Nanosciences et de Nanotechnologies, 91120 Palaiseau, France \\ ${ }^{2}$ IMEC, Kapeldreef 75, B-3001 Leuven, Belgium
}

(Dated: January 6, 2021)

\begin{abstract}
We show experimentally through single-shot time-resolved conductance measurements that magnetization reversal through domain wall motion in sub-100 $\mathrm{nm}$ diameter magnetic tunnel junctions is dominated by two distinct stochastic effects. The first involves the incubation delay related to domain wall nucleation, while the second results from stochastic motion in the Walker regime. Micromagnetics simulations reveal several contributions to temporal pinning of the wall near the disc center, including vertical Bloch line nucleation and wall precession. We show that a reproducible ballistic motion is recovered when Bloch and Néel wall profiles become degenerate in energy in optimally sized discs, which enables quasi-deterministic motion.
\end{abstract}

The reversal of magnetization in nanostructures is a challenging problem for fundamental studies and technological applications. Beyond the coherent reversal mode in which all moments precess in unison as the magnetization switches, which is predicted [1-3] for lateral dimensions below $\approx 25 \mathrm{~nm}$ but rarely observed in practice [4-6], the possibility of intermediate states involving nonuniform magnetic textures makes quantitative prediction of switching thresholds and durations a difficult task. The issue is exacerbated at finite temperatures, where thermal fluctuations render the reversal process stochastic. This is particularly problematic for information storage applications, where deterministic switching is sought [7].

For perpendicularly-magnetized thin film discs with diameters greater than $25 \mathrm{~nm}$, the reversal mode following the minimum energy path is predicted to involve the nucleation and propagation of a domain wall (DW) [8-12]. Such modes are therefore subject to stochastic effects in both the process of nucleation, which generally occurs at edge boundaries, and in the process of propagation as the wall sweeps across the nanostructure. Since dipolar fields are nonuniform across such finite-sized systems, thermal fluctuations can induce a variety of phenomena that can transform the wall structure during reversal.

In this research article, we present experimental evidence of strong stochastic contributions to the free layer reversal in circular magnetic tunnel junctions under SpinTransfer Torques ([13], STT). Time-resolved measurements are interpreted with the aid of micromagnetics simulations and analytical modeling, which show that large-angle precession of the magnetization within the wall, and the nucleation of vertical Bloch lines can contribute to temporal pinning effects observed. We show how such effects can be mitigated when Bloch and Néel wall structures become degenerate in energy; this suppresses most of the variability in the wall propagation dynamics.

\footnotetext{
* thibaut.devolder@u-psud.fr
}

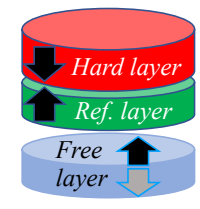

(a)

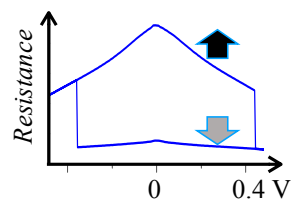

(b)

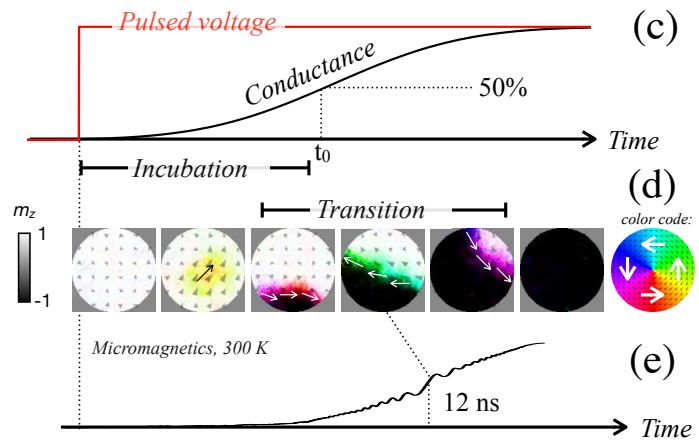

Figure 1. a): Device geometry. b) Resistance versus Voltage hysteresis loop. c) Sketch of the experimental procedure. d) Snapshots of some micromagnetic configurations during the simulated reversal of a $80 \mathrm{~nm}$ device at $300 \mathrm{~K}$ for a spin current of $5.4 \times 10^{10} \mathrm{~A} / \mathrm{m}^{2}$. (e) Corresponding time-resolved magnetic moment.

\section{EXPERIMENTAL METHODS}

The devices studied are presented in Fig. 1(a): they are $\mathrm{CoFeB} / \mathrm{MgO}$-based magnetic tunnel junctions (MTJs) with reference and hard layers organized in a standard synthetic antiferromagnet configuration. The MTJ switches by STT at zero field [Fig. 1(b)]. It is specifically optimized $[14,15]$ to ensure easy domain wall propagation within the free layer. The primary requirement on the free layer material is to avoid non-uniformities that would perturb the domain wall dynamics. The structural non-uniformities - potentially inducing pinning sites- are best avoided when the free layer growth can be optimized 
by a proper freedom on the chosen buffer. Therefore we used free layers embedded in top-pinned MTJs instead of bottom pinned MTJs. The non-uniformities can also arise from the non-uniform stray field of the reference layers of the MTJ. To minimize the detrimental effect of these unavoidably non-uniform [16] stray fields, we work with free layer possessing a low moment (hence low Zeeman energy density) as well as large thickness, which ends by a cap of $8 \AA$ of $\mathrm{FeCoB}$ in contact with the $\mathrm{MgO}$ tunnel oxide. These stringent requirements lead to MTJs that exhibit modest transport properties, with a magneto-resistance typically of $80 \%$ for a resistance-area product of $R A=9.6 \Omega . \mu \mathrm{m}^{2}$. In addition, the materials' trade-offs made to achieve nice wall-propagative free layers lead to a reduced stability of the reference layers ; this impedes a caveat-free experimental study of the parallel to antiparallel ( $\mathrm{P}$ to $\mathrm{AP}$ ) transition such that the experimental part of this research article shall focus on the sole AP to $\mathrm{P}$ switching. The experimental focus is on a device of diameter $100 \mathrm{~nm}$, typical of the behavior in the interval of investigated sizes $(70-100 \mathrm{~nm})$.

Our set-up applies fast rising voltage steps with maximally flat plateaus and time-resolves the device conductance [Fig. 1(c)] in a single shot manner ; the experimental configuration is very similar to the one described ref. [14]. Note that the device impedances are much greater than the characteristic impedance of its surroundings, such that changes in the device conductance during switching do not change the applied voltage. The timeresolved conductance curves are thus illustrative of the device dynamics at constant applied voltage. In response to the voltage step, the device incubates during a variable delay and then its conductance changes abruptly. We fit the conductance waveforms with the ansatz $\operatorname{erf}\left[\left(t-t_{0}\right) / \tau\right]$ to define an incubation delay $t_{0}$ and a transition time $\tau$, which is thus the $24-76 \%$ rise time. No correlation was ever detected between the incubation delays and the transition times. Micromagnetic simulations indicate that the transition corresponds to the sweeping of a DW through the device [Fig. 1(d)].

\section{TIME-RESOLVED SWITCHING}

Results from time-resolved switching are shown in Fig. 2. If the pulse polarity is chosen to induce the AP to $\mathrm{P}$ switching, the conductance first rises gradually in the first 5 ns after the pulse onset. Experiments at lower voltage (not shown) indicate that in fact the conductance approaches asymptotically an on-current higher value, likely as a consequence of Joule heating. This interpretation is corroborated by the fact that no such conductance rise is observed for the other voltage polarity when the pulse is applied on the $\mathrm{P}$ state, whose conductance is notably temperature independent. The subsequent timeevolution of the conductance reflects the magnetic moment $\left\langle m_{z}\right\rangle$ of the free layer, with noticeable fluctuations of the incubation delay and of the transition time. Two distinct switching regimes are observed, depending of the magnitude of STT. At high bias [Fig. 2(a)], the conductance waveforms are monotonic, with ns-scale incubation delays and transition times. In the given example, the incubation lasts in average $\left\langle t_{0}\right\rangle=5.6 \mathrm{~ns}$ and is slightly skewed to higher values [Fig. 2(b)]. Conversely, the distribution of transition times is rather symmetric about its average value $\langle\tau\rangle=2.5 \mathrm{~ns}$ [Fig. 2(c)].

When reducing the STT to just above the quasistatic switching threshold (low bias regime), the dynamics slows down while getting more complex, particularly when the conductance is nearly midway between the initial and final states [Fig. 2(d)]. The distribution of transition times becomes asymmetric. Three types of switching events can be identified. In most events (black curves), the conductance still evolves monotonically with transition times typically of 3 to $4 \mathrm{~ns}$. We will see that these "ballistic" curves correspond to when the DW sweeps rather regularly through the device. For a fraction of events (20\% probability, red curves), a pronounced oscillation is observed: the conductance first passes above the midway value, then it decreases for $1.3 \pm 0.1 \mathrm{~ns}$ until it resumes rising. The transition time is longer, typically 4 to 6 ns. We shall refer to these events as "central oscillation" events as we will see that they arise when the wall oscillates once about the disc center. Finally, in rare occasions $(\approx 2 \%$ probability, green curves) the midway conductance is crossed multiple times and $\tau$ exceeds 7 ns. These events shall be referred to as the "multipleswing" ones that occur when a 180 degree vertical Bloch line [17] appears in the wall while it is slow in the central region of the disc. These interpretations are based on the forthcoming micromagnetic simulations (Fig. 3).

\section{MICROMAGNETIC SIMULATIONS}

Our simulations use the mumax3 code which solves the Landau-Lifshitz-Gilbert equation using the finite difference method [18] with thermal fluctuations [19]. The $2 \mathrm{R}=80-\mathrm{nm}$ diameter, 2 -nm thick circular disc is discretized with $96 \times 96 \times 1$ cells, in a material [11] of magnetization $M_{s}=1.2 \mathrm{MA} / \mathrm{m}$, exchange stiffness $A_{\text {ex }}=20 \mathrm{pJ} / \mathrm{m}$, perpendicular anisotropy constant $K_{u}=$ $1.18 \mathrm{MJ} / \mathrm{m}^{3}$ and damping $\alpha=0.01$. STT is accounted for by a symmetric Slonczewski term, where we have assumed unit spin polarization for simplicity.

A typical simulated reversal event at $300 \mathrm{~K}$ was presented in Fig.1(d): it starts by the growth of a fluctuating droplet of precessing moments, which leads to nucleation of a 180-degree arc-like curved wall when the droplet encounters the disc edge. The in-plane magnetization within the wall is rather uniform. The wall then drifts across the device by a fast back-and-forth oscillatory motion that is reminiscent [20] of the wall precessional motion occurring above the Walker breakdown. As illustrated by the histograms of Fig. 3e and Fig. 2f, the three categories of transition regimes within the switch- 


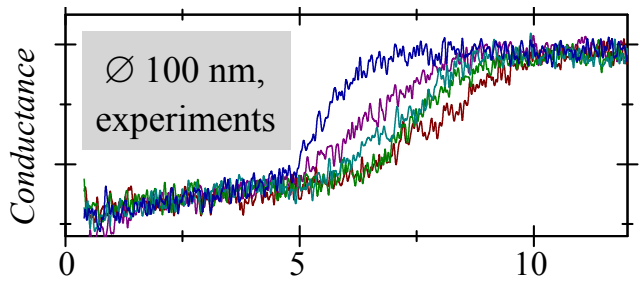

(a)

Time after pulse onset (ns)

High bias

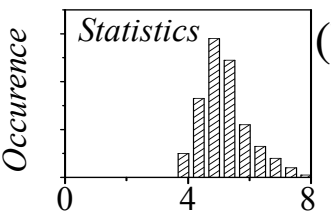

(b)

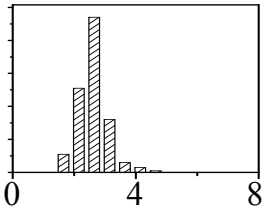

(c)

Incubation delay (ns) Transition time (ns)

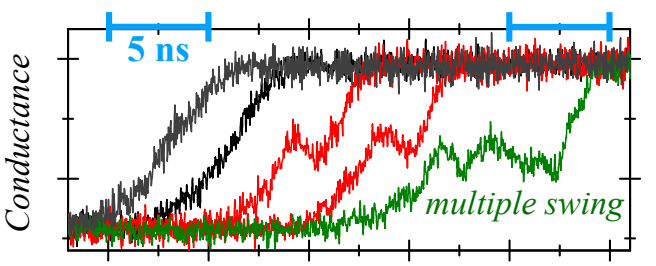

Time relative to switching ( $\mathrm{ns}$ )

Low bias
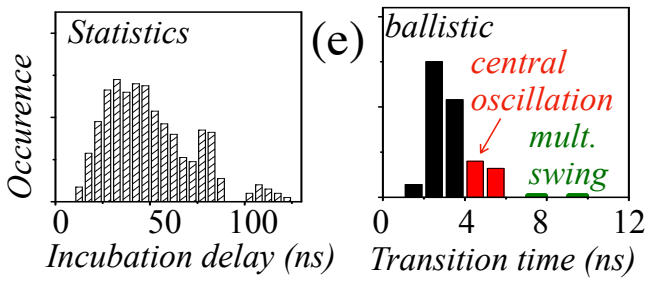

(f)

Figure 2. Experimental signatures of the switching as a result of pulses of (a-c): $630 \mathrm{mV}$ and (d-f): $500 \mathrm{mV}$ on a $100 \mathrm{~nm}$ device. At low bias [panel d)] the curves are horizontally offset to remove incubation delay $t_{0}$ and reveal the three classes of switching events: ballistic crossing of the midway conductance (black), crossing with one single pronounced oscillation (red) and multiple swing-like crossing (green). (e) and (f) statistics of the incubation delay and transition time over 400 switching events ; the two distributions show no correlation.

ing curves found experimentally are reproduced with similar probabilities in the simulations at $300 \mathrm{~K}$ - in contrast the statistics of the incubation delay (Fig. 3d and Fig. 2e) cannot be well reproduced as the shortest time scales, probably because the device temperature is far from constant during the first few nanoseconds as a result of current-induced heating - ; Unfortunately, the corresponding micromagnetic configurations occurring once a wall is nucleated comprise a plethora of active fluctuators that conceal the intrinsic dynamics: on top of the wall motion, there is a breathing of its curvature and a non-uniform beating of its width, as well as ubiquitous short range fluctuations. The physics of the transition is best revealed with $\mathrm{T}=0$ simulations of the wall dynam-
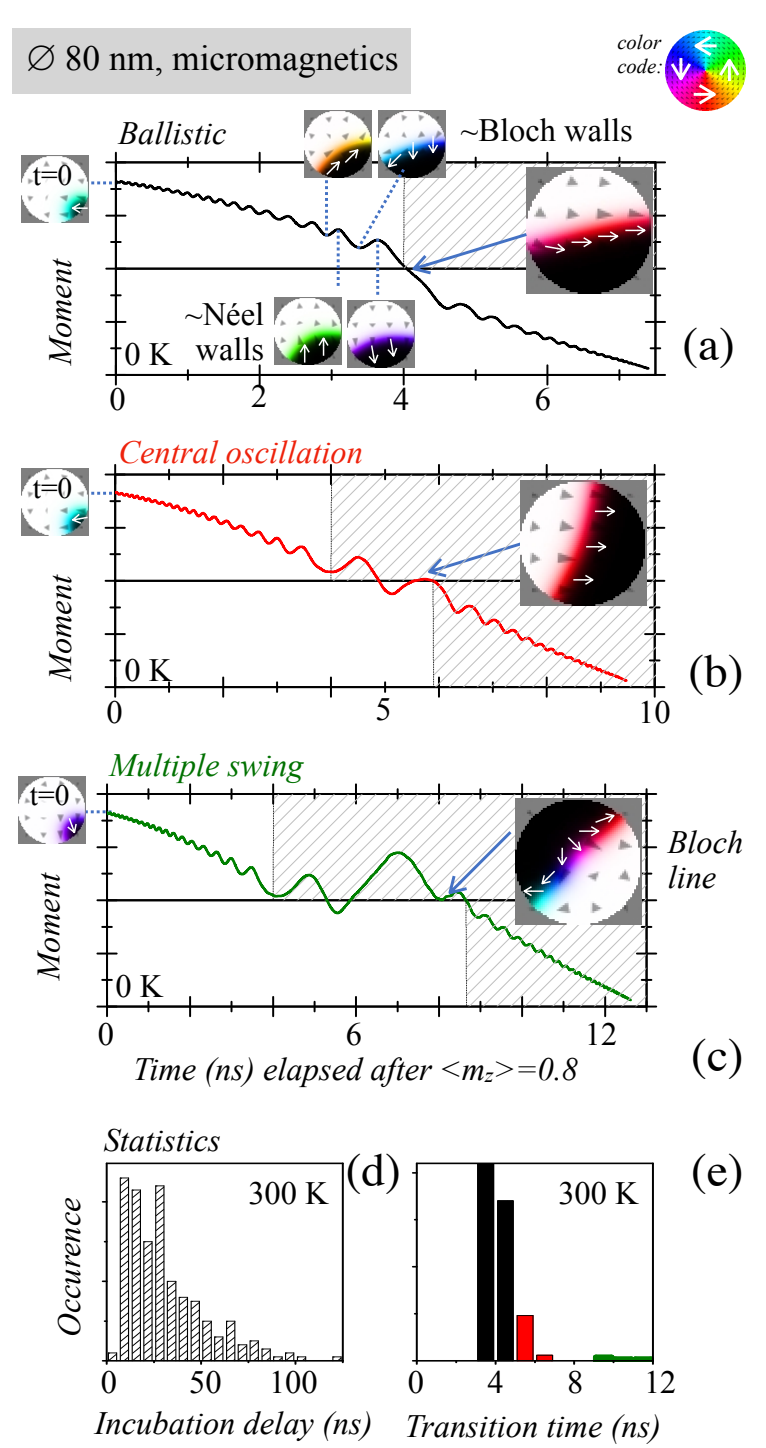

(e)

Figure 3. Simulated DW dynamics within an $80 \mathrm{~nm}$ disc in a low bias situation. (a-c): Examples of dynamics at $\mathrm{T}=0 \mathrm{~K}$ for 3 initial states that differ only in the in-plane projection of the wall magnetization: the tilts at the wall centers are 90, 94 and 128 degrees. The dashed areas underline the switching times; the white arrows sketch the magnetization direction within the wall. (d) and (e): Statistics of the incubation delays and transition times for 300 switching simulations at $\mathrm{T}=300 \mathrm{~K}$ at $3.5 \times 10^{10} \mathrm{~A} / \mathrm{m}^{2}$.

ics. This requires properly chosen initial configurations in which the less relevant fluctuators are intentionally relaxed. We thus designed empirically adequate initial states by numerically placing an optimally bent [9] wall near a disc edge. The wall is set with a uniform inplane magnetization projection, in qualitative line with the $\mathrm{T}=300 \mathrm{~K}$ simulations.

Fig. 3(a-c) illustrates the possible motions of a wall at 0 $\mathrm{K}$ : while drifting, the wall acquires a straight shape when at the center and then bends again. Coincidently with its 


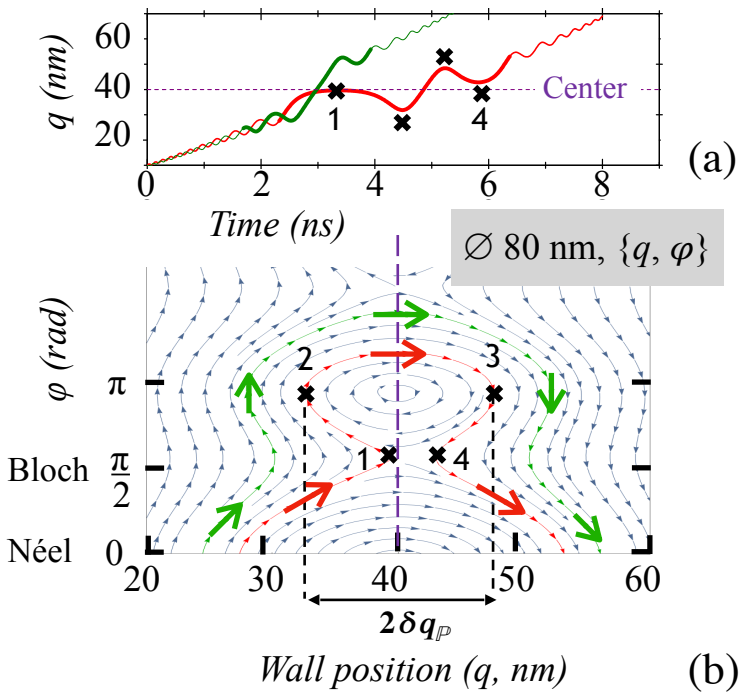

Figure 4. Domain wall dynamics within a $80 \mathrm{~nm}$ disc in the $\{q, \phi\}$ model at low bias $\left(4.5 \times 10^{10} \mathrm{~A} / \mathrm{m}^{2}\right)$. a) Wall trajectories for walls initialized at $q=10 \mathrm{~nm}$ with initial tilts of 48 deg. (green) and $91 \mathrm{deg}$. (red). b) Corresponding trajectories in the $\{q, \phi\}$ space. The red contour between the labels $1,2,3$ and 4 is very close to the frontier of the retention pond $\mathbb{P}$.

velocity modulation, the wall passes periodically from the Bloch to the Néel configuration. Notably, it never crosses the center while in the pure Néel state. The oscillation of the moment is both more pronounced and slower when the wall approaches the disc center. Fig. 3(a-c) are meant to reveal how minute changes in the initial state alter substantially the dynamics when the wall later arrives in the center. Its motion can either be ballistic [Fig. 3(a)], or the wall can fail to cross the center at once. In this case it withdraws before passing the center and resuming its oscillatory drift motion [Fig. 3(b)]. Alternatively, if choosing on purpose a specific initial tilt for which the wall happens to be maximally slow at the disc center, then the non-uniformity of the tilt can grow and lead to [inset in Fig. 3(c)] the creation of a vertical Bloch line [17]. Coincidently, the DW swings multiple times in the central area with a gyration move before finally passing.

\section{COLLECTIVE COORDINATE MODEL FOR THE WALL PROPAGATION DYNAMICS}

Further insight can be gleaned by an extension to the one-dimensional $\{q, \phi\}$ model [21] of wall dynamics, where the motion is parametrized entirely by the position $q$ of a straight wall and the internal wall angle $\phi$ (the "tilt"), which describes the chirality (Bloch or Néel) of the wall structure. The extension comprises accounting for the spatially non-uniform potential for the wall dynamics, which captures the fact that the disc center appears as an energy barrier to overcome, where the barrier height is related to the additional cost in domain wall
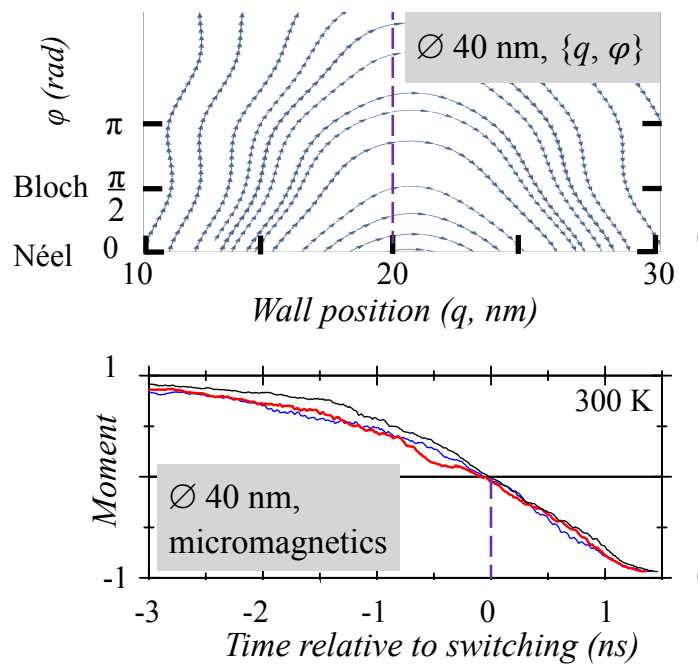

(b)

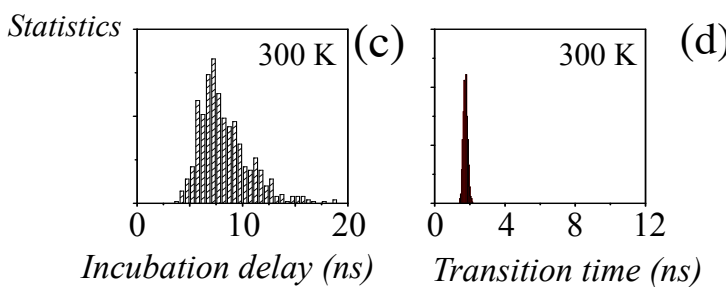

Figure 5. Domain wall dynamics within a $40 \mathrm{~nm}$ disc at $5.4 \times 10^{10} \mathrm{~A} / \mathrm{m}^{2}$. (a) phase portrait of the wall trajectories within the $\{q, \phi\}$ model. (b) Three representative micromagnetic simulations at $300 \mathrm{~K}$. Corresponding histograms of the incubation delay (c) and of the transition time (d).

energy required to extend it laterally across the disc. The equations of motion become:

$$
\begin{aligned}
-\dot{\phi}+\alpha \frac{\dot{q}}{\Delta} & =-\gamma_{0}\left[H_{\mathrm{d}}(q)+H_{\mathrm{str}}(q, \phi)\right] \\
\frac{\dot{q}}{\Delta}+\alpha \dot{\phi} & =\gamma_{0} \frac{H_{N \leftrightarrow B}}{2} \sin 2 \phi+\sigma j,
\end{aligned}
$$

where $\pi \Delta$ is the wall width and $\sigma j$ the magnitude of STT [11]. $\quad H_{d}$ is the stray field of the two domains, $H_{N \leftrightarrow B}$ (typically $30 \mathrm{mT}$ ) is the in-plane field that would be needed to transform a Bloch DW into a Néel DW [22]. To describe the energy barrier in $q$ and $\phi$ directions, we have defined the two contributions to the wall stretch field:

$$
H_{\mathrm{str}}(q, \phi)=\left(H_{k}^{\mathrm{eff}}+\frac{H_{N \leftrightarrow B}}{2} \cos ^{2} \phi\right) \frac{\Delta}{\ell_{\mathrm{DW}}} \frac{\partial \ell_{\mathrm{DW}}}{\partial q}
$$

where $\ell_{\mathrm{DW}}(q)=\frac{1}{\Delta} \int_{-R}^{R} \mathrm{~d} x \operatorname{sech}^{2}\left(\frac{x-q}{\Delta}\right) \sqrt{R^{2}-q^{2}}$ is the effective length of the wall.

Fig. 4(b) shows the phase portrait of the resulting wall trajectories in the $(q, \phi)$ space. The largely vertical flows, which takes the wall from one disc edge to the other, recall that the wall motion is in the Walker regime, since many oscillations of the wall tilt $\phi$ accompany the increase in $q$. The portrait also shows that the number 
of oscillations undergone by the wall is sensitive to the initial wall tilt. This sensitivity induces a stochasticity when the thermal fluctuations drive transitions between neighboring trajectories. Closer to the disc center, we observe that the Néel states $(\phi=0[\pi])$ are associated with energy maxima, while Bloch states $(\phi=\pi / 2[\pi])$ give rise to saddle points. This representation evidences that in addition to the "switching" trajectories (i.e. walls passing from one edge to the other) there is another family of trajectories. Indeed if placing a Néel wall at the center, it is transiently held there. It needs to spiral out of this energy maximum through a lossy back-and-forth transfer of energy between communicating vessels: the position degree of freedom of the wall and the tilt degree of freedom. The region in which walls of proper tilt are transiently pinned is a "retention pond", written $\mathbb{P}$ and illustrated in Fig. 4(b). In the conservative limit, the spiraling-out trajectories inside the retention pond transform into closed circles ; the half size of the pond $\mathbb{P}$ is then:

$$
\delta q_{\mathbb{P}} \approx R \sqrt{H_{N \leftrightarrow B} / H_{\mathrm{k}, \mathrm{eff}}}
$$

\section{REPRODUCIBILITY OF THE TRANSITION TIME AND CONCLUSIONS}

The concept of $\mathbb{P}$ is useful to understand the statistics of the transition time: the DW drift and its oscillation are rather independent phenomena, such that two situations can occur stochastically as a wall heads to the disc center. If the wall avoids the vicinity of $\mathbb{P}$ [green curve in Fig. 4], the $\{q, \phi\}$ model predicts a ballistic curve. If in contrast the wall happens to tangent the pond (red curve), it bypasses it by performing a back-and-forth motion with two pauses at either sides near the disc center [positions 1 and 4 in Fig. 4(b)], in the formerly identified "central oscillation" case. The $\{q, \phi\}$ models can thus explain some of the sensitivity to the tilt. However since the $\{q, \phi\}$ model posits a uniform tilt, it cannot account for the multiple swing trajectories that occur when the wall gets slow enough for a Bloch line to develop therein. The swing-like crossing can only be described using higher dimensionality simulations like in the micromagnetic ones, or a more general collective coordinate model that would include a gradient of the tilt along the wall length. Despite its approximations, the $(q, \phi)$ model is illustrative since it explains why the probability of non-ballistic transition is correlated with the size of $\mathbb{P}$.

If one aims at a reproducible DW propagation while having no handle on the wall tilt, as in the presence of thermal noise, a solution is to find a geometry without retention pond. In discs there exists a single "magic" diameter for which the pond disappears and all wall trajectories are predicted ballistic irrespective of the wall tilt. This happens when the energies of centered walls of Bloch or Néel characters are degenerate, i.e. when $H_{N \leftrightarrow B}=0$. With our material parameters, this diameter is $40 \mathrm{~nm}$ [Fig. 5(a)]. One may object that this is not sufficient to guarantee reproducibility of the transition time, since the $\{q, \phi\}$ model posits a uniform tilt and hence cannot account for the rare "multiple swing" trajectories that occur when the wall gets slow enough for a Bloch line to develop therein.

Fortunately, when using this diameter the wall does not slow down near the disc center: this drastically reduces the probability that fluctuations can pile up and lead to the formation of a Bloch line within the wall. This optimistic conjecture was confirmed with micromagnetics [Fig. 5(b-d)]. While the incubation delays are still distributed for a diameter of $40 \mathrm{~nm}$, the transition regimes exhibit very little variance and the distribution of transition times is particularly narrow: the magic diameter ensures a repeatable wall motion independent from the tilt dynamics and immune from its fluctuations. If implemented jointly with the strategies ensuring reliable nucleation [23-26] or/and reproducible wall tilt and wall position [27], this strategy opens the route for reproducible switching times, which is of interest for memory applications in which write error rates could be substantially lowered.

\section{ACKNOWLEDGMENT}

This work was supported by IMEC's Industrial Affiliation Program on STT-MRAM devices.
[1] J. Z. Sun, Spin-current interaction with a monodomain magnetic body: A model study, Physical Review B 62, $570(2000)$.

[2] W. H. Butler, T. Mewes, C. K. A. Mewes, P. B. Visscher, W. H. Rippard, S. E. Russek, and R. Heindl, Switching Distributions for Perpendicular Spin-Torque Devices Within the Macrospin Approximation, IEEE Transactions on Magnetics 48, 4684 (2012).

[3] H. Tomita, S. Miwa, T. Nozaki, S. Yamashita, T. Nagase, K. Nishiyama, E. Kitagawa, M. Yoshikawa, T. Daibou, M. Nagamine, T. Kishi, S. Ikegawa, N. Shimomura,
H. Yoda, and Y. Suzuki, Unified understanding of both thermally assisted and precessional spin-transfer switching in perpendicularly magnetized giant magnetoresistive nanopillars, Applied Physics Letters 102, 042409 (2013).

[4] J. Z. Sun, S. L. Brown, W. Chen, E. A. Delenia, M. C. Gaidis, J. Harms, G. Hu, X. Jiang, R. Kilaru, W. Kula, G. Lauer, L. Q. Liu, S. Murthy, J. Nowak, E. J. OSullivan, S. S. P. Parkin, R. P. Robertazzi, P. M. Rice, G. Sandhu, T. Topuria, and D. C. Worledge, Spin-torque switching efficiency in $\mathrm{CoFeB}-\mathrm{MgO}$ based tunnel junctions, Physical Review B 88, 104426 (2013). 
[5] C. Hahn, G. Wolf, B. Kardasz, S. Watts, M. Pinarbasi, and A. D. Kent, Time-resolved studies of the spintransfer reversal mechanism in perpendicularly magnetized magnetic tunnel junctions, Physical Review B 94, 214432 (2016).

[6] T. Devolder, A. Le Goff, and V. Nikitin, Size dependence of nanosecond-scale spin-torque switching in perpendicularly magnetized tunnel junctions, Physical Review B 93, 224432 (2016).

[7] A. V. Khvalkovskiy, D. Apalkov, S. Watts, R. Chepulskii, R. S. Beach, A. Ong, X. Tang, A. Driskill-Smith, W. H. Butler, P. B. Visscher, D. Lottis, E. Chen, V. Nikitin, and M. Krounbi, Basic principles of STT-MRAM cell operation in memory arrays, Journal of Physics D: Applied Physics 46, 074001 (2013).

[8] C.-Y. You, Switching current density reduction in perpendicular magnetic anisotropy spin transfer torque magnetic tunneling junctions, Journal of Applied Physics 115, 043914 (2014).

[9] G. D. Chaves-OFlynn, G. Wolf, J. Z. Sun, and A. D. Kent, Thermal Stability of Magnetic States in Circular Thin-Film Nanomagnets with Large Perpendicular Magnetic Anisotropy, Physical Review Applied 4, 024010 (2015).

[10] J. Sampaio, A. V. Khvalkovskiy, M. Kuteifan, M. Cubukcu, D. Apalkov, V. Lomakin, V. Cros, and N. Reyren, Disruptive effect of Dzyaloshinskii-Moriya interaction on the magnetic memory cell performance, Applied Physics Letters 108, 112403 (2016).

[11] P. Bouquin, S. Rao, G. S. Kar, and T. Devolder, Size dependence of spin-torque switching in perpendicular magnetic tunnel junctions, Applied Physics Letters 113, 222408 (2018).

[12] L. Desplat and J.-V. Kim, Entropy-reduced Retention Times in Magnetic Memory Elements: A Case of the Meyer-Neldel Compensation Rule, Physical Review Letters 125, 107201 (2020).

[13] J. C. Slonczewski, Currents, torques, and polarization factors in magnetic tunnel junctions, Physical Review B 71, 024411 (2005).

[14] T. Devolder, O. Bultynck, P. Bouquin, V. D. Nguyen, S. Rao, D. Wan, B. Sore, I. P. Radu, G. S. Kar, and S. Couet, Back-hopping in Spin-Transfer-Torque switching of perpendicularly magnetized tunnel junctions, arXiv:2006.05108 [cond-mat, physics:physics] (2020), arXiv: 2006.05108 .

[15] T. Devolder, J. V. Kim, J. Swerts, S. Couet, S. Rao, W. Kim, S. Mertens, G. Kar, and V. Nikitin, Material Developments and Domain Wall-Based NanosecondScale Switching Process in Perpendicularly Magnetized STT-MRAM Cells, IEEE Transactions on Magnetics 54, 1 (2018).

[16] T. Devolder, R. Carpenter, S. Rao, W. Kim, S. Couet, J. Swerts, and G. S. Kar, Offset fields in perpendicularly magnetized tunnel junctions, Journal of Physics D: Ap- plied Physics 52, 274001 (2019).

[17] V. Krizakova, J. P. Garcia, J. Vogel, N. Rougemaille, D.deSouza. Chaves, S. Pizzini, and A. Thiaville, Study of the velocity plateau of Dzyaloshinskii domain walls, Physical Review B 100, 214404 (2019).

[18] A. Vansteenkiste, J. Leliaert, M. Dvornik, M. Helsen, F. Garcia-Sanchez, and B. V. Waeyenberge, The design and verification of MuMax3, AIP Advances 4, 107133 (2014).

[19] J. Leliaert, J. Mulkers, J. De Clercq, A. Coene, M. Dvornik, and B. Van Waeyenberge, Adaptively time stepping the stochastic Landau-Lifshitz-Gilbert equation at nonzero temperature: Implementation and validation in MuMax3, AIP Advances 7, 125010 (2017).

[20] T. Devolder, J.-V. Kim, F. Garcia-Sanchez, J. Swerts, W. Kim, S. Couet, G. Kar, and A. Furnemont, Timeresolved spin-torque switching in $\mathrm{MgO}$-based perpendicularly magnetized tunnel junctions, Physical Review B 93, 024420 (2016).

[21] A. Thiaville, Y. Nakatani, J. Miltat, and N. Vernier, Domain wall motion by spin-polarized current: a micromagnetic study, Journal of Applied Physics 95, 7049 (2004).

[22] A. Mougin, M. Cormier, J. P. Adam, P. J. Metaxas, and J. Ferr, Domain wall mobility, stability and Walker breakdown in magnetic nanowires, Europhysics Letters 78, 57007 (2007).

[23] S. Pizzini, J. Vogel, S. Rohart, L.D. Buda-Prejbeanu, E. Jue, O. Boulle, I.M. Miron, C.K. Safeer, S. Auffret, G. Gaudin, and A. Thiaville, Chirality-Induced Asymmetric Magnetic Nucleation in $\mathrm{Pt} / \mathrm{Co} / \mathrm{AlOx}$ Ultrathin Microstructures, Physical Review Letters 113, 047203 (2014).

[24] B. Lacoste, M. M. de Castro, T. Devolder, R. C. Sousa, L. D. Buda-Prejbeanu, S. Auffret, U. Ebels, C. Ducruet, I. L. Prejbeanu, L. Vila, B. Rodmacq, and B. Dieny, Modulating spin transfer torque switching dynamics with two orthogonal spin-polarizers by varying the cell aspect ratio, Physical Review B 90, 224404 (2014).

[25] O. Bultynck, M. Manfrini, A. Vaysset, J. Swerts, C. J. Wilson, B. Soree, M. Heyns, D. Mocuta, I. P. Radu, and T. Devolder, Instant-On Spin Torque in Noncollinear Magnetic Tunnel Junctions, Physical Review Applied 10, 054028 (2018).

[26] S. Finizio, K. Zeissler, S. Wintz, S. Mayr, T. Weels, A. J. Huxtable, G. Burnell, C. H. Marrows, and J. Raabe, Deterministic Field-Free Skyrmion Nucleation at a Nanoengineered Injector Device, Nano Letters 19, 7246 (2019).

[27] M. Baumgartner, K. Garello, J. Mendil, C. O. Avci, E. Grimaldi, C. Murer, J. Feng, M. Gabureac, C. Stamm, Y. Acremann, S. Finizio, S. Wintz, J. Raabe, and P. Gambardella, Spatially and time-resolved magnetization dynamics driven by spinorbit torques, Nature Nanotechnology 12, 980 (2017), number: 10. 